\title{
Voxar Labs
}

Veronica Teichrieb, Francisco Simões, Lucas Figueiredo, João Paulo Lima, João Marcelo Teixeira, Rafael Roberto, Alana da Gama, Thiago Chaves, Arlindo Gomes, Maria Euzébio, and Voxar Labs team

Centro de Informática - Universidade Federal de Pernambuco

http://www.cin.ufpe.br/voxarlabs

\{vt, fpms, lsf, jpsml, jmxnt, rar3, aefg, tmc2, agsn, mlte\}@cin.ufpe.br

\begin{abstract}
Voxar Labs is a research group focused in augmenting experiences through research, innovation and collaboration with academia and industry. It develops cutting-edge multi-disciplinary research in the large area of Spatial Computing, tackling the inner areas of Extended Reality, Computer Vision and Natural Interaction. The laboratory aims to create impact through R\&D\&I, technology transfer, scientific publications, patents and human-resources formation. It is one of the most productive Augmented Reality research groups in the Latin America, also being recognized with seven best papers and ten first-place competitions' prizes over the nine years of its existence. Voxar Labs is part of the Informatics Center of the Federal University of Pernambuco, located in Recife - Pernambuco, Brazil.
\end{abstract}

Keywords: extended reality; augmented reality; spatial computing; computer vision; natural interaction.

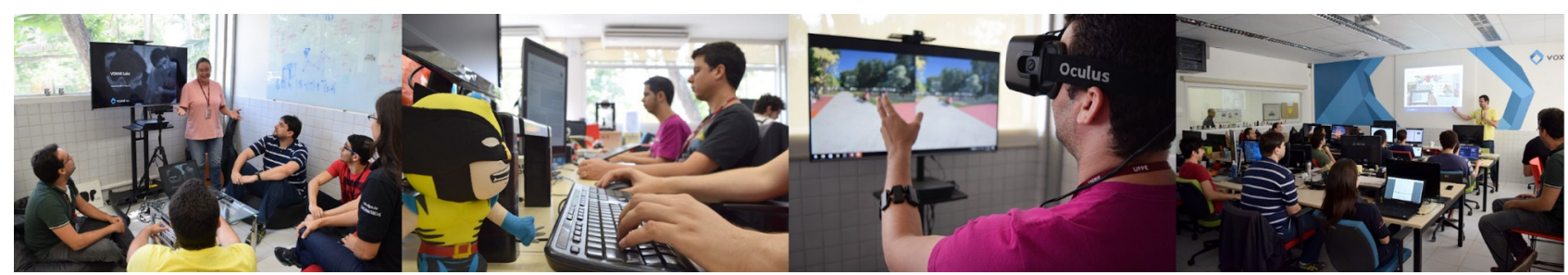

Figure 1. Voxar Labs collaborators and laboratory headquarters at CIn-UFPE.

\section{Introduction}

Voxar Labs [1] is a research group founded in 2011, which develops research, development and innovation in the fields of Spatial Computing, with focus on Extended Reality (AR, MR, VR), Computer Vision and Natural Interaction. The name is coined as a reference to these areas, being a junction of "Voxel", as the fundamental unit of a spatial world that we want to build or understand, and Augmented Reality acronym "AR".

The Voxar Labs mission is to develop people by augmenting experiences. Based on this mission, its projects and research challenges arise from a problem focused environment. The values representing its core culture principles are creativity, cooperation, reliability, responsibility, flexibility, and enjoyment.

The group leadership arises from a network of professors and researchers, from UFPE and other institutions from Pernambuco, headed by Dr. Veronica Teichrieb (lab director and associate professor at CInUFPE), and includes Dr. Francisco Simões (Federal Institute of Pernambuco - IFPE), Dr. João Paulo Lima (Federal Rural University of Pernambuco - UFRPE), Dr. João Marcelo Teixeira (Electronics and Systems Department of UFPE), Dr. Lucas Figueiredo (Senior Research Scientist), Dr. Rafael Roberto (Senior Research Scientist), and Dr. Alana Da Gama (Biomedical Engineering Department of UFPE). This partnership reinforces the interdisciplinary importance for the group, in addition to establishing important alliances with other research institutions from the state.
The Voxar Labs team also includes one postdoctoral research fellow, one Ph.D. researcher, seven Ph.D. candidates, five master's students and 18 undergraduate students. There are a total of 48 members, including professors, researchers, engineers, Ph.D. candidates, students (MSc. BSc.), and staff from computer sciences and other fields, such as design, education, and physiotherapy. Some of these members can be seen in Figure 1, as well as the current headquarters of the lab.

Despite being relatively young, the lab has already won several prizes, including seven best papers (five from SVR), and ten first-place competitions' prizes. The laboratory also contains the only researcher from Latin America to appear in the list of the top 160 authors in Augmented Reality (AR) worldwide, Veronica Teichrieb. More information about Voxar Labs is presented in the following sections.

\section{Surroundings and context (State, City and University)}

The state of Pernambuco, in the northeast coast of Brazil, is famous for its beaches and cultural attractions. In addition to its continental area, it holds the Fernando de Noronha archipelago, a natural site on UNESCO's World Heritage list. It also unites culture and development, being one of the leading technology centers in the country.

The capital of Pernambuco is Recife. It is the oldest capital in Brazil, maintaining a set of mansions from colonial times that are still preserved, especially in its downtown area (Figure 2). Recife is famous for its 
strong culture, with dance, manual arts, theater, and cinema. People are very friendly and welcoming, sharing a unique passion for their hometown.

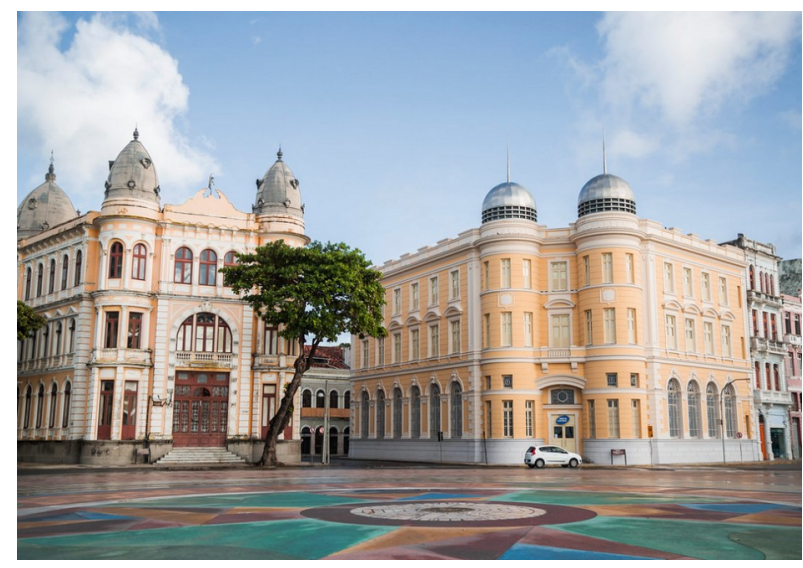

Figure 2. Recife downtown area with preserved colonial buildings. The area holds Porto Digital, an important technological park for Latin America

In the last 15 years, the city has come to have a great national and international prominence as an important hub for IT technology and creative economy. Much of this is due to Porto Digital [2], one of the most important technological parks in Latin America in terms of revenue and number of companies.

The main university in Pernambuco is the Universidade Federal de Pernambuco (UFPE), with its main campus located in Recife (Figure 3). Founded in 1946, UFPE aggregates more than 40 thousand people, including teachers, staff, and students. It ranks 10th nationwide and 45th in Latin America according to QS World University Rankings [3]. Rankings such as CSRankings point the Center of Informatics of UFPE in the South American leadership in the last 3 years in scientific production in the areas of Visualization, Robotics and Artificial Intelligence, among others, with UFPE in 5th position.

Among its centers, one that stands out is the Center of Informatics (CIn-UFPE), one of the most renowned centers in Brazil and Latin America (Figure 4). The CInUFPE Graduate Program stands out among the seven best in Brazil, and its courses include academic master's and Ph.D. programs, professional master's and PhD programs, and specializations. Also, the Program has the title of "Center of Excellence and Reference" in teaching and research, with national and international renown, achieving the grade "7" (the highest) in the evaluation of the Brazilian Coordination for the Improvement of Higher Education Personnel (CAPES). In this field, CIn-UFPE maintains a significant and growing production, with the annual publication of about 40 articles in indexed international journals and more than 100 articles in national and international conferences, in addition to books and book chapters.

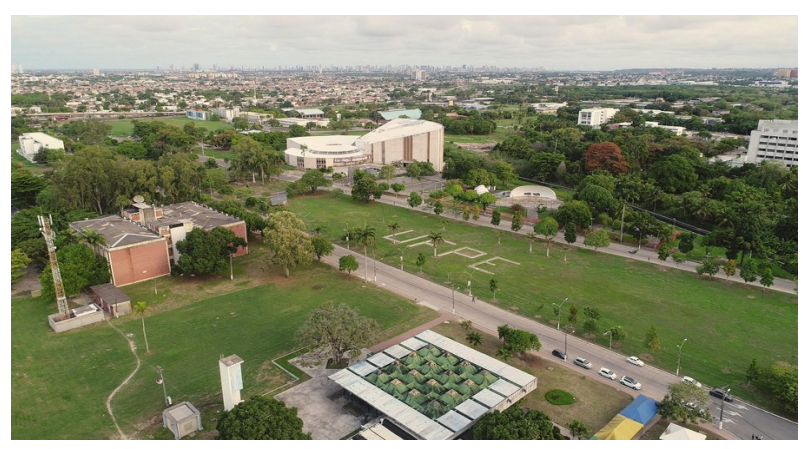

Figure 3. Universidade Federal de Pernambuco (UFPE).

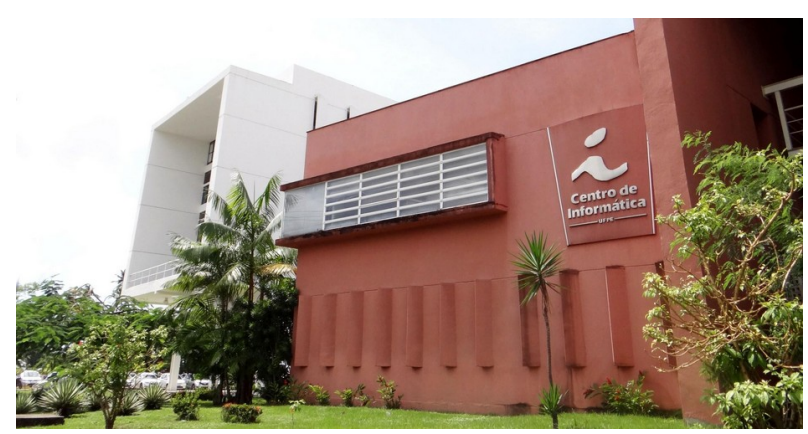

Figure 4. Center of Informatics of the UFPE (Cin-UFPE).

\section{Representative projects}

Voxar labs uses an YouTube account (youtube.com/user/ voxarlabs) to showcase its main results in research, development and innovation [4]. As examples of the lab effort in long term research, two are very representative: the 3D Tracking area and the Sicure project (Figure 5). The 3D Tracking research area has powered international cooperation projects (i.e., INRIA/UChile/ UFPE and TUGraz/Kyushu/UFPE), industry projects and patents, various collaborative PostDoc and Ph.D.'s (e.g., with Microsoft Research, EPFL, TUGraz), research papers and prizes in high quality venues (ISMAR [5][6], ICRA [7], C\&G [8][9], IJCNN[10], Sensors [11]).

The project called Sicure (former Ikapp project), shows how the interaction of real-world challenges and multidisciplinary applied research can produce impactful results, focusing on at home safe physiotherapy. This project boosted an international cooperation (TUMunich), PhD.s and master's, multidisciplinary cooperation with physiotherapy and biomechanical engineering researchers, a spin-off creation, and many high-quality papers and prizes (C\&G [12], 3DUI [13]).

\section{Innovation through research}

Amongst the development strategies adopted by research laboratories, the Voxar Labs approaches the research activity as a means to achieve innovation, and therefore deliver impactful results to the society. The core vision is to bridge the gap between research findings on Spatial Computing (from our works and others') and its applications. 


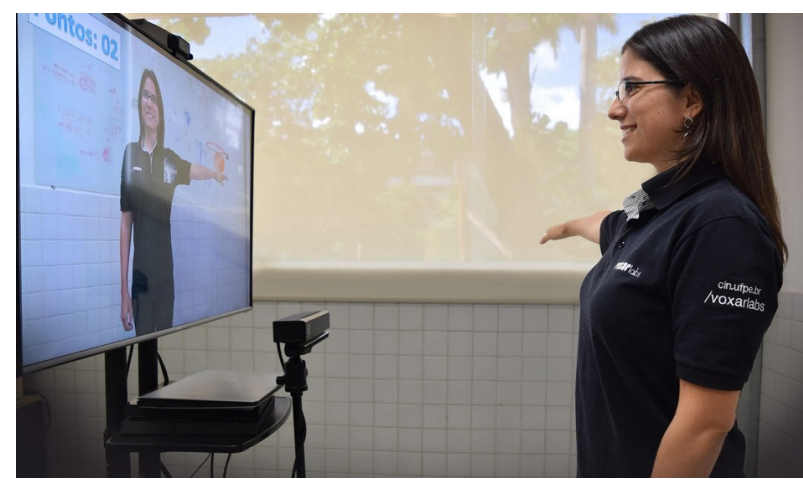

Figure 5. Sicure project (former Ikapp project).

This way, research methodologies within the Voxar Labs include innovation strategies, tailored Design Thinking processes to identify relevant use case scenarios and partners, to then solve problems within our society. For instance, internal processes such as the Foresight method (iftf.org/foresightessentials/), developed by Institute for the Future (IFTF), are conducted to steer the research towards non-trivial problems that in some cases may even not be yet revealed. Therefore, the approach of conception and development of researches is twofold: reactive, receiving demands from industry partners; but also proactive, analyzing exposed needs in society, and forecasting future opportunities.

Initiatives conducted in this direction include hosting hackathons, using as base ready-to-transfer technologies from the research, publicizing the contributions, and fostering the creation of competitive startups from the start, given the advanced technical background that will be used as core for their products (Figure 6). At last, the Voxar Labs also works with innovation hubs (e.g., SOFTEX - softexrecife.org.br), hosting events to promote collaboration with local and national industry partners. Grounded on developed models for technology transfer and licensing, the laboratory elaborates and engages on match-making strategies to relate companies needs to families of technologies developed by the lab, enabling partners to tackle unsolvable problems otherwise.

\section{Social, technological and scientific contributions}

In recent years, the Voxar Labs has stood out for carrying out high impact research, having, in the year 2019 alone, published five articles in indexed international journals, 12 papers in national and international conferences, generated two patents, and is in the process of creating its first two spin-offs. This is the result of research done by its members, as well as partnerships with companies and other universities, including HP, Samsung, Harvard GSE, Université de Montréal, Kyushu University, to name only a few.

Since its foundation, Voxar Labs won the best paper in five of the last eight editions of SVR, which is the main Brazilian event in the area. Among the awards, some highlights are the first place in the @Home category of the Latin American and Brazilian Robotics
Competition - LARC/CBR, and the first place in the Offsite Tracking Competition of the IEEE ISMAR, both in 2015. Voxar Labs also won innovation prizes such as the Sports Hack Brazil of the IBM Hackathon (held at Campus Party Recife), Layar creation challenge in 2011, Metaio Developer Contest in 2013, and competitions at SVR and the Brazilian Symposium on Computer Games and Digital Entertainment (SBGames).

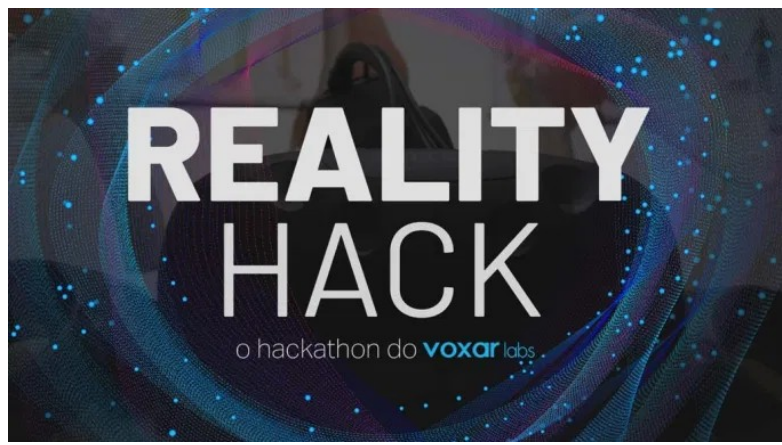

Figure 6. Hackathon promoted by the Voxar Labs to give opportunity to the birth of startups with cutting-edge technologies.

In a recent review [14], prof. Mark Billinghurst systematically searched Scopus papers to find the top 160 authors in Augmented Reality (AR) and the institutions that they come from (Figure 7).

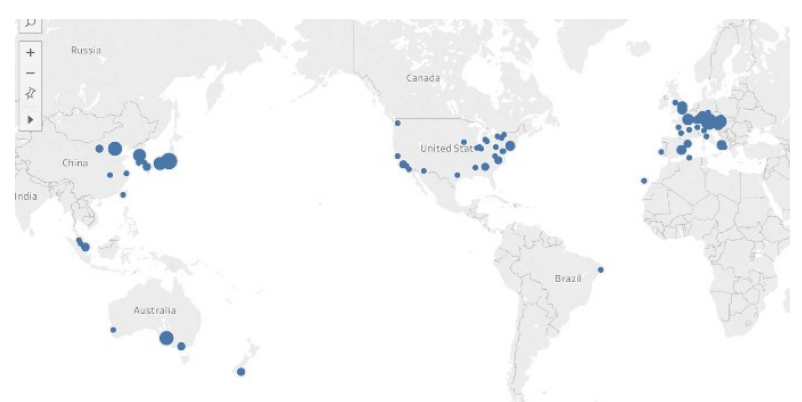

Figure 7. Where in the world is AR Research Happening? Medium publication from prof. Mark Billinghurst in 2018 [14].

The list is based on the number of publications produced by each author. Veronica Teichrieb is the only researcher from Latin America to appear in that list. When using the same criteria for Latin America, 5 from the top 10 researchers belong to the Voxar Labs team.

In 2020, Voxar Labs CIn-UFPE is proud to organize IEEE Symposium on Mixed and Augmented Reality (ISMAR) [15]. In addition, the three major Brazilian conferences in VR/AR, CG/CV and Digital Games. These conferences, sponsored by the Brazilian Computer Society (SBC) are the Symposium on Virtual and Augmented Reality (SVR) [16], the Conference on Graphics, Patterns and Images (SIBGRAPI) [17] and the Symposium on Games and Digital Entertainment (SBGames) [18]. All conferences were planned to be hosted in the Recife area before COVID-19. However, to preserve the safety and well-being of all participants, they are going to be held as virtual conferences. The Voxar labs team can be seen in Figure 8. 


\section{References}

[1] Voxar Labs. https://www.cin.ufpe.br/voxarlabs. Accessed on 8/10/2020.

[2] Porto Digital. https://www.portodigital.org/. Accessed on 8/10/2020.

[3] QS Latin American University Rankings 2020. https://www.topuniversities.com/university-rankings/latinamerican-university-rankings/2020. Accessed on 8/10/2020.

[4] Voxar Labs youTube channel. https://www.youtube.com/user/voxarlabs.

[5] Roberto, R.; Lima, J. P.; Araújo, T.; Teichrieb, V. (2016) Evaluation of motion tracking and depth sensing accuracy of the tango tablet. In: Proc. 2016 IEEE International Symposium on Mixed and Augmented Reality (ISMARAdjunct), p. 231-234. IEEE. DOI: 10.1109/ismaradjunct.2016.0082

[6] Lima, J. P., Uchiyama, H., Teichrieb, V., \& Marchand, E. (2012) Texture-less planar object detection and pose estimation using depth-assisted rectification of contours. In: Proc. 2012 IEEE International Symposium on Mixed and Augmented Reality (ISMAR), p. 297-298. IEEE. DOI: 10.1109/ISMAR.2012.6402582

[7] Olivier, N.; Uchiyama, H.; Mishima, M.; Thomas, D.; Taniguchi, R. I.; Roberto, R. et al. (2018) Live structural modeling using RGB-D SLAM. In: Proc. 2018 IEEE International Conference on Robotics and Automation (ICRA), p. 6352-6358. IEEE. DOI: 10.1109/ICRA.2018.8460973

[8] Roberto, R; Lima, J. P.; Uchiyama, H.; Teichrieb, V.; Taniguchi, R. I. (2019) Geometrical and statistical incremental semantic modeling on mobile devices. Computers \& Graphics 84: 199-211. DOI: 10.1016/j.cag.2019.09.003

[9] Roberto, R.; Lima, J. P.; Teichrieb, V. (2016) Tracking for mobile devices: A systematic mapping study. Computers \& Graphics 56: 20-30. DOI: 10.1016/j.cag.2016.02.002
[10] Felix, H.; Rodrigues, W.; Macedo, D.; Simoes, F.; Oliveira, A.; Teichrieb, V.; Zanchettin, C. (2020) Squeezed Deep 6dof Object Detection Using Knowledge Distillation. In: Proc. 2020 International Joint Conference on Neural Networks (IJCNN). Glasgow. ArXiv:2003.13586

[11] Mishima, M.; Uchiyama, H.; Thomas, D.; Taniguchi, R. I., Roberto; R., Lima, J. P.; Teichrieb, V. (2019) Incremental 3D Cuboid Modeling with Drift Compensation. Sensors 19(1): 178. DOI: 10.3390/s19010178

[12] Cavalcanti, V. C.; de Santana Ferreira, M. I.; Teichrieb, V.; Barioni, R. R.; Correia, W. F. M.; Da Gama, A. E. F. (2019) Usability and effects of text, image and audio feedback on exercise correction during augmented reality based motor rehabilitation. Computers \& Graphics 85: 100-110. DOI: 10.1016/j.cag.2019.10.001

[13] Da Gama, A.; Chaves, T.; Figueiredo, L.; Teichrieb, V. (2012) Improving motor rehabilitation process through a natural interaction based system using kinect sensor. In: Proc. 2012 IEEE Symposium on 3D User Interfaces (3DUI), p. 145-146). IEEE. DOI: 10.1109/3DUI.2012.6184203

[14] Where in the World is AR Research Happening? https://medium.com/@marknb00/where-in-the-world-isar-vr-research-happening-ddebbdc6436b. Accessed on8/10/2020.

[15] ISMAR 2020. http://ismar20.org/. Accessed on $8 / 10 / 2020$.

[16] SVR 2020. https://svr2020.cin.ufpe.br/. Accessed on 8/10/2020.

[17] SIBGRAPI 2020. https://sibgrapi2020.cin.ufpe.br/. Accessed on 8/10/2020.

[18] SBGAMES 2020. https://sbgames2020.cin.ufpe.br/. Accessed on 8/10/2020.

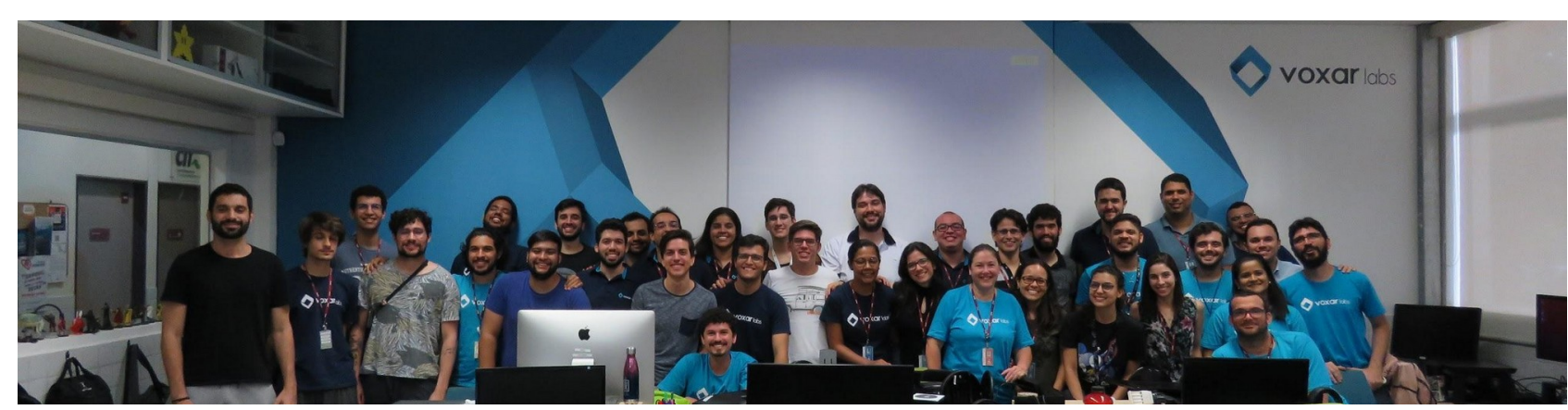

Figure 8. The Voxar Labs team in 2019/2020 closing year meeting. 